Remsperger, Hermann (2004): Inflationsdifferentiale im Euro-Raum: Ursachen und Konsequenzen, Heidelberg

Riese, Hajo/Spahn, Heinz-Peter (1990) (Hg.): Geldpolitik und ökonomische Entwicklung, Studien zur monetären Ökonomie 4, Regensburg

Thomasberger, Claus (1993): Europäische Währungsintegration und globale Währungskonkurrenz, Tübingen

Thomasberger, Claus (1998): , Internationale Wettbewerbsfähigkeit: Sinn und Unsinn einer wirtschaftspolitischen Konzeption, Bonn

Williamson, John/Miller, Marcus 1987: Targets and Indicators: A Blueprint for the International Coordination of Economic Policy, Policy Analysis in International Economics, No. 22, Washington D.C.: Institute for International Economics

\title{
Ein Schritt vor und zwei Schritte zurück? Zur Kritik an den Vorschlägen der Europäischen Kommission zur Strukturförderung nach 2006 Katharina Erdmenger ${ }^{*}$, Astrid Ziegler ${ }^{\circ}$
}

\section{Einleitung}

Im Juli 2004 veröffentlichte die Europäische Kommission ihre Vorschläge für die Verordnungen über den Europäischen Fonds für regionale Entwicklung, den Europäischen Sozialfonds und den Kohäsionsfonds (vgl. Kommission der Europäischen Gemeinschaften 2004), die Grundlage für die Gestaltung der Strukturfonds in der neuen Förderperiode ab 2007 sein werden. Damit ist das europäische Entscheidungsverfahren über die künftige Gestaltung der Strukturförderung eröffnet, es soll im Laufe des Jahres 2005 mit einer Einigung der 25 Mitgliedstaaten und der europäischen Institutionen abgeschlossen werden. Es wäre nun zu vermuten, dass im Mittelpunkt der aktuellen Debatte über die Zukunft der Strukturförderung die von der Kommission vorgeschlagenen Förderinhalte in einer erweiterten EU der 25 stehen würden. Tatsächlich wird diese Diskussion aber um die Frage geführt, wie viel Geld in den europäischen Haushalt für die Strukturförderung eingezahlt werden muss und wie viel die einzelnen Mitgliedstaaten davon erhalten sollen.

EU-Nettozahler - allen voran Deutschland - bestehen darauf, das Volumen ihrer Einzahlungen in den EU-Haushalt insgesamt auf ein Prozent ihrer Wirtschaftsleistung zu begrenzen. ${ }^{\mathrm{I}}$ Die anderen Mitgliedsländer wollen an der bisherigen Regelung (I,I4 Prozent des

* DGB-Bundesvorstandsverwaltung, Berlin.

- Wirtschafts- und Sozialwissenschaftliches Institut (WSI) in der Hans Böckler Stiftung, Düsseldorf.

I EU-Nettozahler, die sich der deutschen Forderung angeschlossen haben, sind Österreich, Frankreich, Großbritannien, die Niederlande und Schweden. 
Bruttonationaleinkommens [BNE]) festhalten. Die mittelfristige Finanzausstattung der EU entscheidet jedoch über die zukünftige Ausrichtung der Europäischen Strukturfonds. Da der größte Haushaltsposten - die Agrarausgaben - bis 2013 fest geschrieben wurde (vgl. Axt 2003) und der Haushaltsansatz anderer Politikfelder (wie z.B. der Forschungspolitik) aufgestockt werden soll, würden sich Kürzungen im EU-Haushalt zwangsläufig auf den zweitgrößten Block, auf die Strukturfonds, auswirken.

Diese Haushaltsverhandlungen überlagern jedoch einen wichtigen Punkt, nämlich, dass es nach der Erweiterung der EU umso notwendiger ist, über den konzeptionellen Rahmen der Europäischen Strukturfonds nach 2006 zu debattieren. Nach Artikel I58 des EG-Vertrages hat die Gemeinschaft die Aufgabe, "eine harmonische Entwicklung der Gemeinschaft als Ganzes« zu fördern und »die Unterschiede im Entwicklungsstand der verschiedenen Regionen und den Rückstand der am stärksten benachteiligten Gebiete oder Inseln, einschließlich der ländlichen Gebiete, zu verringern«. Dies wird auch in der EUVerfassung im Artikel I-3 betont und herausgestellt, dass die EU »den wirtschaftlichen, sozialen und territorialen Zusammenhalt und die Solidarität unter den Mitgliedstaaten « ${ }^{2}$ fördert. Mit Hilfe der Europäischen Strukturfonds sollen diese Ziele erreicht werden. Bisher baute das System der europäischen Strukturpolitik auf der Solidarität zwischen den strukturstarken und -schwachen Regionen auf. Dieser ausgleichspolitische Ansatz konzentriert die strukturpolitischen Hilfen auf die strukturschwächsten Gebiete Europas. Die eingesetzten Wirkungskontrollen attestierten dieser Politik durchaus positive Effekte. ${ }^{3}$ Diesen Evaluierungsstudien steht aber der 2003 veröffentlichte Sapir-Bericht gegenüber, der der Europäischen Kommission vorschlägt, die Europäischen Strukturfonds im Sinne der Lissabon-Strategie unter wachstumsorientierten Aspekten einzusetzen und diese auf die Wachstumszentren in der EU zu konzentrieren (vgl. André Sapir Groupe 2003). ${ }^{4}$ Diese inhaltliche Neuorientierung hat im Kommissionsvorschlag deutlichen Niederschlag gefunden, wie noch zu zeigen sein wird.

Nicht nur auf europäischer Ebene wird heftig über die zukünftigen Europäischen Strukturfonds gestritten, auch in Deutschland treffen unterschiedliche Koalitionen aufeinander (vgl. Ziegler 2003a). Blieben die heutigen Abgrenzungskriterien für die struktur-

2 EU-Amtsblatt, C 310, 47. Jahrgang, I6. Dezember 2004.

3 So zeigte z.B. die Zwischenevaluierung zum deutschen Ziel I-Gebiet für 2000 bis 2003 , dass "die Strukturfondsinterventionen (in Ostdeutschland) [...] ein Potential (besitzen), das dazu führt, dass nach Ende der Förderperiode (2006) das Niveau des Sozialprodukts dauerhaft um ungefähr I \% höher liegt als ohne deren Einsatz (GEFRA et al. 2004: 15).

4 Ziel des Berichts der Expertengruppe unter Vorsitz von André Sapir sollte sein, die Auswirkungen der beiden strategischen wirtschaftspolitischen Ziele zu analysieren, die sich die EU für das zoIo auslaufende Jahrzehnt gesetzt hat: die Union zum wettbewerbsfähigsten und dynamischsten wissensbasierten Wirtschaftsraum mit dauerhaftem Wirtschaftswachstum und größerem sozialen Zusammenhalt zu machen (die sog. "Lissabon-Agenda «) und die bevorstehende Erweiterung durch eine rasche Hebung des Lebensstandards in den neuen Mitgliedstaaten zum Erfolg werden zu lassen. Die Gruppe sollte das gesamte System der EU-Wirtschaftspolitiken überprüfen und eine Strategie zur Steigerung des Wachstums bei gleichzeitiger Sicherung von Stabilität und Zusammenhalt in einer erweiterten EU vorschlagen. 
schwachen Regionen ${ }^{5}$ nach Ziel I und Ziel 2 erhalten, gäbe es ab 2007 in Westdeutschland kaum mehr eine Ziel-2-Region und einige Regionen in den neuen Bundesländern würden ihren Ziel-I-Förderstatus verlieren. In Verbindung mit dem Subventionsvorbehalt der EU hätte Deutschland somit einen eingeschränkten Handlungsspielraum für eigene strukturpolitische Interventionen, und dies in Zeiten, in denen die Strukturprobleme in Ost und West zunehmen und strukturpolitische Maßnahmen eher ausgebaut statt abgebaut werden müssten.

Anders als in den vergangenen Reformdebatten zur Zukunft der Europäischen Strukturfonds geht es diesmal also nicht nur um eine inhaltliche Weiterentwicklung in nicht in Frage gestellten Bestandteilen, sondern um die Grundstrukturen der europäischen Integrationspolitik und des Solidaritätsgedankens innerhalb der Europäischen Strukturfonds. Vor diesem Hintergrund hat sich der vorliegende Beitrag zum Ziel gesetzt, die Kommissionsvorschläge einer zukünftigen Kohäsionspolitik zu analysieren. Dazu werden diese sowie der aktuelle Stand der Debatte dargestellt. Dem schließt sich eine Bewertung der Kommissionsvorschläge an; es soll aufgezeigt werden, welche Konsequenzen die Umsetzung der Kommissionsvorschläge für Europa und für Deutschland hätte.

\section{Die Vorschläge der Europäischen Kommission}

Die zukünftigen Europäischen Strukturfonds ${ }^{6}$ stehen im Zeichen von zwei großen Projekten: der Integration der zehn neuen Mitgliedsländer in die EU und der Zielsetzung der Lissabon-Strategie (vgl. dazu auch Fußnote 4). Bei der Umsetzung beider Projekte fällt den Europäischen Strukturfonds eine Schlüsselposition im Hinblick auf die Finanzierung dieser Projekte zu - dies wird durch den Kommissionsvorschlag deutlich.

Für den Zeitraum von 2007 bis 20I3 - die Laufzeit der kommenden Strukturfondsperiode - will die Kommission rund ein Drittel des EU-Haushalts für die Kohäsionspolitik bereitstellen: In der von ihr vorgesehenen und erhofften Größenordnung des EU-Haushaltes wären dies rund 336,3 Milliarden $€$. Dies entspräche ca. o,4I Prozent des BNE der EU. Wenn man die Ausgaben für die ländliche Entwicklung und die Fischereiwirtschaft, die ab 2007 der Agrarmarktpolitik zufallen sollen, hinzuaddiert, binden die gesamten Strukturinterventionen 0,46 Prozent des BNE der EU. Nach den Kommissionsvorstellungen werden diese Mittel je zur Hälfte auf die neuen und alten Mitgliedstaaten entfallen, wobei drei Viertel des Gesamtbetrages für die Regionen und Mitgliedstaaten mit Entwicklungsrückstand vorgesehen werden sollen.

Die Europäische Kommission will in Zukunft die Strukturfonds auf drei Ziele konzentrieren (vgl. auch Abbildung I, S. 28f.):

5 Ziel I: Entwicklung und strukturelle Anpassung der Regionen mit Entwicklungsrückstand; Ziel 2: Wirtschaftliche und soziale Umstellung von Gebieten mit Strukturproblemen.

6 Einen Überblick über das aktuelle Konzept der Europäischen Strukturfonds gibt Ziegler (2003b: $20 \mathrm{ff}$.). 
- Ziel I: Konvergenz und Wettbewerbsfähigkeit

Mit dem Ziel »Konvergenz und Wettbewerbsfähigkeit« soll die bisherige Ziel-I-Förderung als zentraler Bestandteil der EU-Strukturfonds fortgesetzt werden. Unter dieses Ziel sollen alle Regionen fallen, die weniger als 75 Prozent des BIP/Kopf in der EU-25 aufweisen. In diese Ziel-Kategorie werden auch diejenigen Regionen aufgenommen, die auf Grund des statistischen Effektes ${ }^{7}$ aus der Ziel-I-Förderung herausfallen würden. Die vom statistischen Effekt der Erweiterung betroffenen Regionen erhalten im Rahmen einer Phasing-out-Phase eine Beihilfe, die mit der Zeit immer mehr abnehmen wird. Nach aktuellen Berechnungen der Kommission würden alle ostdeutschen Regionen in die neue Ziel-I-Förderung fallen. ${ }^{8}$ Darüber hinaus sollen diejenigen Mitgliedstaaten, deren Pro-Kopf-BNE weniger als 90 Prozent des Gemeinschaftsdurchschnitts beträgt, über den Kohäsionsfonds in den Bereichen Verkehr und Umwelt gefördert werden. Diesem Ziel I sollen ungefähr 78,5 Prozent der Finanzmittel zufallen.

- Ziel 2: Regionale Wettbewerbsfähigkeit und Beschäftigung

Die Europäische Kommission tritt des Weiteren dafür ein, dass es auch nach 2006 eine Förderung jenseits der Ziel-I-Förderung geben soll. Sie hat in ihren Vorschlägen ein neues Ziel 2 geschaffen, das die derzeitigen Ziele 2 und 3 zusammenfasst. Inhaltlich soll sich das neue Ziel 2 auf zwei Pfeiler stützen. Der erste regionale Pfeiler soll den strukturellen Wandel in industriellen, städtischen und ländlichen Gebieten fördern und voran bringen. Im Bereich der regionalen Wettbewerbsfähigkeit geht es in erster Linie um die Unterstützung der Lissabon-Strategie und hauptsächlich um die Förderung von Innovation und Wissensgesellschaft. Allerdings hat die Kommission nicht definiert, was unter diesen Schlagworten ihrer Meinung nach förderfähig wäre. Der zweite national orientierte Pfeiler, der sich an der europäischen Beschäftigungsstrategie orientiert, soll sich auf Maßnahmen zur Förderung von Vollbeschäftigung, Arbeitsplatzqualität, Arbeitsproduktivität und soziale Integration konzentrieren. Nach den Vorstellungen der Kommission soll das neue Ziel 2 in die Verantwortung der Mitgliedstaaten übergehen, die in Bezug auf den ersten Pfeiler selbst die förderwürdigen Regionen auswählen. Dieses Ziel soll ungefähr 17,2 Prozent der gesamten Strukturfondsmittel binden.

7 Durch die wirtschaftliche und soziale Lage in den neuen Mitgliedsländern wird der EU-Durchschnitt bei den relevanten Abgrenzungskriterien sinken. Dadurch werden aufgrund des statistischen Effektes ab 2007 viele heutige Förderregionen des Ziel-I-Gebietes aus der Förderung heraus fallen, ohne dass sich an ihrer eigenen wirtschaftlichen Situation etwas Grundlegendes verändert hätte.

8 Die Berechnung bezog sich auf den Durchschnitt der Jahre 1999-2000-200I in der EU-I5 (Europäische Kommission 2004: I90 ff.). Bei dieser Berechnung wird außerdem deutlich, dass sich die westdeutschen Regionen in den letzten Jahren weiter auseinander entwickelten. Unter anderem liegt der Regierungsbezirk Lüneburg mit 76,6 Prozent des durchschnittlichen BIP pro Kopf der EUI5 nur knapp über der Grenze (75 Prozent), um in die Ziel-I-Kategorie zu fallen. Die endgültige Abgrenzung für die neue Förderperiode wird voraussichtlich im Jahr 2005 auf Basis der Jahre 2002, 2003 und 2004 erfolgen. 


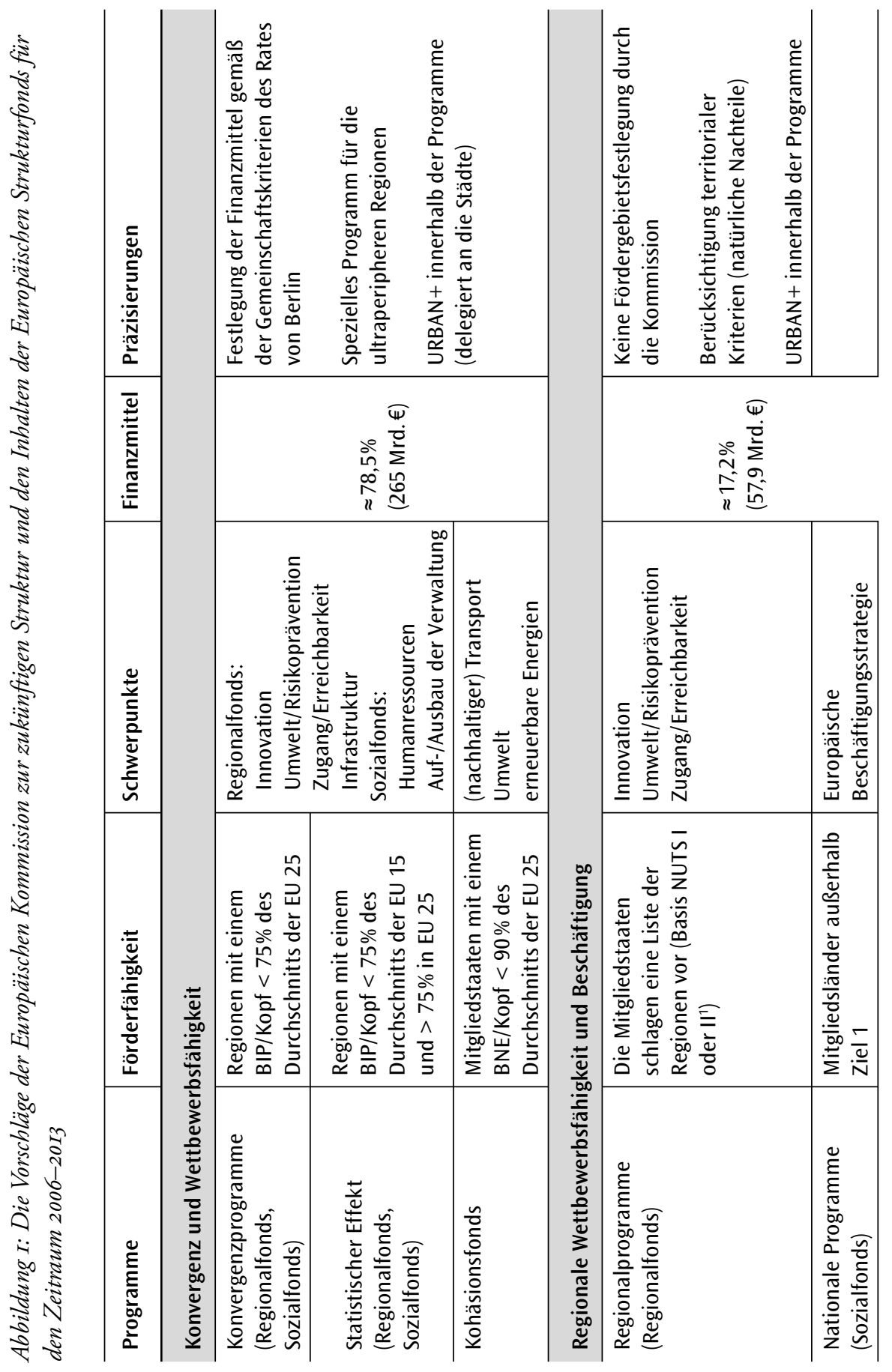




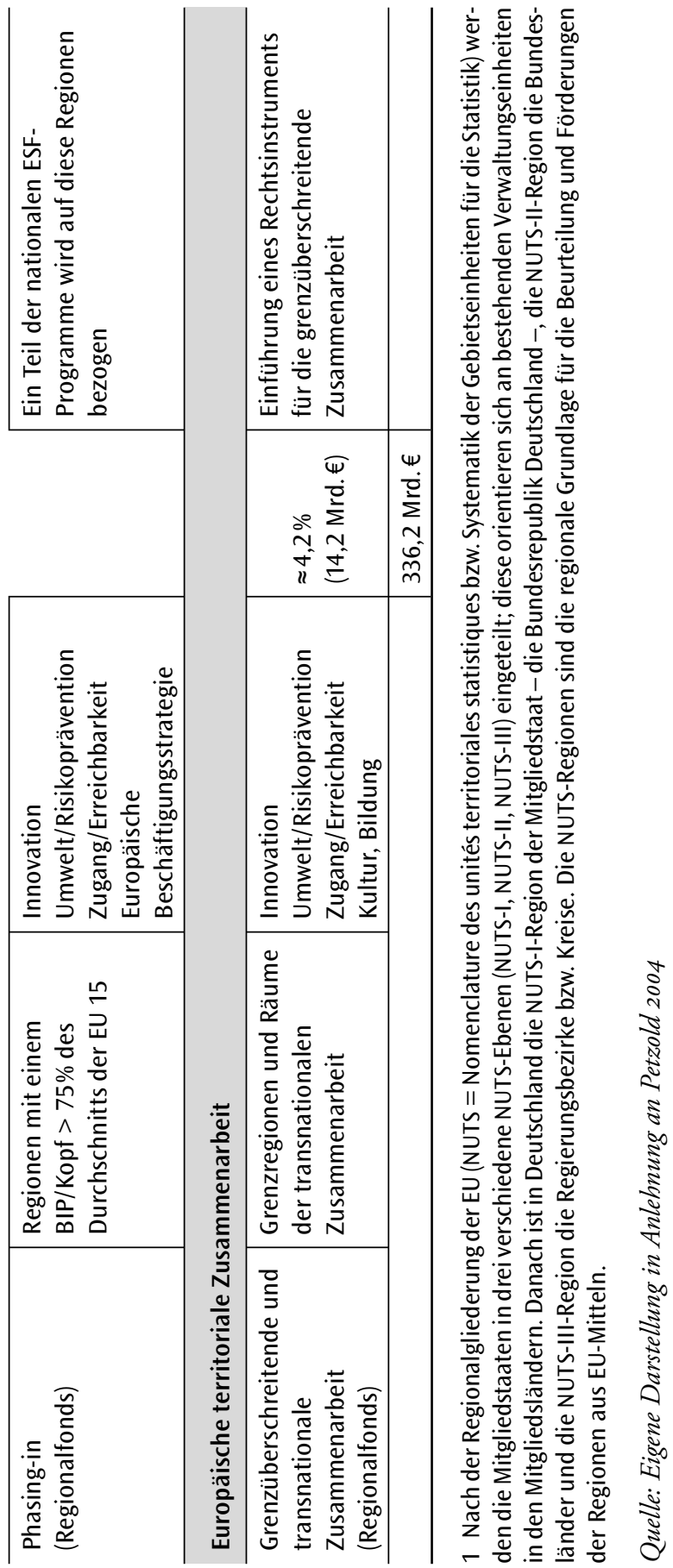




\section{- Ziel 3: Europäische territoriale Zusammenarbeit}

Das dritte Ziel - die europäische territoriale Zusammenarbeit - setzt an dem Erfolg der laufenden Gemeinschaftsinitiative INTERREG an und betrifft die Förderung von Grenzregionen der transnationalen Zusammenarbeit, der interregionalen Zusammenarbeit und der grenzüberschreitenden Zusammenarbeit an den Außengrenzen der EU. Für die zukünftige grenzüberschreitende Zusammenarbeit soll ein neues Rechtsinstrument geschaffen werden, das in Form einer grenzübergreifenden Regionalbehörde arbeiten und die bestehenden Reibungsverluste, die auf Grund der administrativen und rechtlichen Unterschiede an den Grenzen entstehen, bewältigen soll. Für dieses Ziel sind 4,2 Prozent der Mittel reserviert.

Die Grundsätze der Kohäsionspolitik (strategische Planung, mehrjährige Programmierung, dezentrale Verwaltung, systematische Begleitung und Bewertung, Partnerschaft, Zusätzlichkeit und Kofinanzierung, geteilte Verantwortung) werden durch den Kommissionsvorschlag bestätigt. Die seit langem geäußerte Kritik der Überbürokratisierung greift die Kommission in der Form auf, dass sie ausschließlich Monofonds-Programme vorschlägt und damit in Zukunft für ein Ziel jeweils nur noch ein Fonds zuständig wäre. Des Weiteren will sie die Stufen der Programmierung auf zwei reduzieren, so dass neben den Entwicklungsplänen auf regionaler Ebene nur noch ein politisches Dokument auf Ebene des Mitgliedstaates angefertigt werden müsste.

\section{Aktueller Stand der Diskussion}

Die Haltung der deutschen Bundesregierung zu den Kommissionsvorschlägen lässt sich knapp zusammenfassen: „So wenig einzahlen wie möglich - so wenig Vorgaben von der Kommission wie möglich." Von ihrem Ziel einer Deckelung der Einzahlungen in den EU-Haushalt bei einem Prozent des nationalen BNE ist die Bundesregierung bisher nicht abgewichen. Zudem zeigt sie wenig Sympathie für die "nationalen Strategiepapiere«, am liebsten würde sie offensichtlich die mit der Umsetzung der Strukturfonds verbundene Planung allein den Ländern überlassen - und damit ein wichtiges Koordinierungsinstrument aus der Hand geben. Zwar ist es in Anbetracht des Zeithorizontes verständlich, dass man den Planungsaufwand reduzieren will - wenn 2007 mit der Förderung nach den neuen Regelungen begonnen werden soll, muss die Planung Ende 2006 abgeschlossen sein. Die Bundesregierung steht mit ihrer Position jedoch alleine da und isoliert sich mit ihrer Auffassung im Kreis der 25 EU-Mitgliedstaaten. Zudem würde eine Gesamtstrategie des Bundes und der Länder die Effizienz des Fondseinsatzes steigern.

Des Weiteren ist die Diskussion in Deutschland geprägt von einer Verhärtung der Positionen des Bundes einerseits und insbesondere der neuen Länder andererseits: Das Interesse der neuen Länder ist es, ihren Ziel-I-Status zu wahren. Das setzt eine Einzahlung des Bundes in den EU-Haushalt voraus, die höher ist als das eine Prozent des BNE, auf die der Bund sich festgelegt hat. Aus der Perspektive der neuen Bundesländer kommt die Haltung des Bundes einer Absage an eine nachhaltige Strukturentwicklung gleich, zumal die restriktive Haltung bei den EU-Fonds mit weiteren Einschränkungen bei der bundes- 
deutschen Regionalpolitik einhergeht. Aufgrund der unterschiedlichen Bund-Länder-Positionen hat sich - anders als in den vorangegangenen Reformen - der Bundesrat mit der Europäischen Strukturförderung beschäftigt und dazu einen Beschluss verabschiedet. ${ }^{9}$ Hierin spiegeln sich die Interessenlagen der alten und der neuen Bundesländer deutlich wider: Die Fortführung der Ziel-I-Förderung auch in den Phasing-out-Regionen wird begrüßt, für die Förderung von Regionen im neuen Ziel 2 wird eine Abgrenzung nach klaren sozio-ökonomischen Kriterien verlangt. Die ehemaligen Ziel-2-Regionen in den alten Bundesländern wollen sich ihren Anteil an der Förderung sichern. Alle Bundesländer haben sich damit gegen die von der Bundesregierung geforderte Beschränkung der Gesamtfördersumme gewandt.

\section{Bewertung der Kommissionsvorschläge}

Der Kommissionsvorschlag stellt einen Versuch dar, die Förderung von strukturschwachen Gebieten und die Förderung von Problemgruppen des Arbeitsmarktes mit der Wachstumsorientierung der Lissabon-Strategie in der Kombination von Ziel I und Ziel 2 miteinander zu verbinden.

Jedoch steht und fällt der Vorschlag der Kommission mit dem Finanzetat. Obwohl im Vorfeld der Kommissionsvorschläge die Nettozahlerländer eine Erhöhung des EU-Haushalts ausgeschlossen haben, geht die Kommission von einer Erhöhung der Finanzmittel für die Strukturfonds gegenüber der laufenden Förderperiode aus, und zwar von 245 Milliarden $€$ in der Förderperiode 2000-2006 (Eltges 2003: Io) auf 336,3 Milliarden $€$ für den Zeitraum 2007-2013. Sollte sich die Europäische Kommission in den weiteren Verhandlungen mit ihrer Vorstellung für den Gesamthaushalt nicht durchsetzen können, würde eine Kürzung des strukturpolitischen Etats voll zu Lasten der bisherigen Fördergebiete in den alten Mitgliedsländern gehen, da ein breiter politischer Konsens besteht, die Europäischen Strukturfonds prioritär in den strukturschwächsten Regionen - also in den neuen Mitgliedsländern und für die bedürftigsten Personengruppen - einzusetzen. Das neue Ziel 2 könnte damit kaum mehr finanziert werden. Ein solches Szenario ist durchaus vorstellbar, wenn man die Vorschläge zur Strukturpolitik in den Gesamtrahmen der Debatte um den EU-Haushalt einordnet: Auf Grund des erwähnten Sapir-Berichtes sind im Vorschlag der Europäischen Kommission für die EU-Haushalte der Jahre 2007 bis 2013 die Haushaltsposten aufgewertet worden, die der Förderung groß angelegter Investitionsprojekte dienen. Dazu gehören insbesondere die Transeuropäischen Netze (TEN) und die Forschungspolitik. Da politischer Konsens zwischen der Kommission und den Mitgliedstaaten darüber besteht, dass diese Politikfelder der Umsetzung der Lissabon-Strategie unmittelbar dienen, sind Kürzungen dieser Bereiche nicht zu erwarten. Sparpotential käme daher nur für die neue »Priorität 2 « der Strukturförderung in Frage. In diesem Fall würden sich die Europäischen Strukturfonds vollständig auf das Ziel I und auf die Integration der neuen Mitgliedsländer zurückziehen.

9 Beschluss des Bundesrates vom I5.I0.2004, Drucksache 57I/04. 
Aber nicht nur der Finanzrahmen ist ungeklärt, auch die Zieldefinition des neu geschaffenen Ziels 2 mit der zugrunde gelegten Förderphilosophie erscheint unausgegoren. Das neue Ziel 2 setzt sich aus den alten Zielen 2 und 3 zusammen. Mithin fasst es arbeitsmarkt- und regionalpolitische Elemente zusammen. Angesichts der seit langem geführten Diskussion um die Verknüpfung von Arbeitsmarkt- und Regionalpolitik ist diese Zusammenlegung im neuen Ziel 2 zunächst positiv zu bewerten, aber die Struktur des Ziels 2 lässt vermuten, dass es der Kommission weniger darum ging, Synergieeffekte zwischen Arbeitsmarkt- und Regionalpolitik zu fördern, da beide nicht inhaltlich miteinander verknüpft sind. Für den "Ziel 3«-Teil soll es ähnlich wie im bisherigen Ziel 3 nationale Programme geben. Inhaltlicher Schwerpunkt bleibt die Unterstützung der europäischen Beschäftigungsstrategie. Der »Ziel 2«-Teil soll zwar regional programmiert werden, jedoch soll es für die Abgrenzung der förderfähigen Regionen keine nach einheitlichen Kriterien erstellte Gebietskulisse in der EU mehr geben. Den Mitgliedstaaten wäre völlig freie Hand gelassen, in welchen Regionen und für welche Projekte sie ihre Ziel-2-Gelder einsetzen. Das Ziel 2 könnte ab 2007 von Mitgliedstaat zu Mitgliedstaat in sehr unterschiedlicher Weise ausgestaltet sein.

Abgesehen davon, dass fraglich ist, ob ein Mitteleinsatz nach ganz unterschiedlichen Kriterien in den verschiedenen Mitgliedstaaten zur Umsetzung gemeinsamer europäischer Zielsetzungen dient, sollte es bei der Umsetzung der Fonds neben ihrer Beschäftigungswirksamkeit vor allem auf deren innovative Qualität ankommen. Die europäischen Fonds haben in erheblichem Maße zur Modernisierung der deutschen Regional- und Arbeitsmarktpolitik beigetragen (vgl. Reissert 2004). Insbesondere in den I99oer Jahren wurde in Deutschland das breitere und flexiblere Förderspektrum des Regional- und Sozialfonds genutzt. So richteten bspw. die Bundesländer mit Hilfe der EFRE-Mittel landeseigene Förderprogramme und -initiativen ein, die über die klassische Investitionsförderung des nationalen Instrumentes der Gemeinschaftsaufgabe »Verbesserung der regionalen Wirtschaftsstruktur« (GRW) hinausging und vor allem technologie-, umwelt-, verkehrs- und stadtentwicklungspolitische Vorhaben beinhalteten. Auch wurde das GRW-Instrumentarium um die Förderung des Regionalmanagements und um die Erstellung von regionalen Entwicklungskonzepten erweitert. Ebenso wurde das nationale Förderspektrum im Bereich der Arbeitsmarktpolitik um innovative Elemente (wie z.B. die vorbeugende Qualifizierung von Arbeitslosigkeit bedrohter Beschäftigter) ergänzt und Defizite des deutschen Konzeptes beseitigt. Die Förderung von Arbeitslosen, die keine ausreichenden Ansprüche an die Arbeitslosenversicherung besitzen (insbesondere arbeitslose Sozialhilfeempfänger), wurde über Mittel der Strukturfonds realisiert. Es ist zu befürchten, dass diese Ansätze zurückgefahren werden, wenn sie nicht mehr durch die Fonds, sondern alleine durch Bund und Länder initiiert werden sollten.

Außerdem wird an den Vorschlägen zum neuen Ziel 2 deutlich, dass die Orientierung auf die Lissabon-Strategie dann Schwierigkeiten bereiten kann, wenn die Strukturfonds als ergänzendes Element zu einer gesamteuropäischen Investitionsstrategie verstanden werden, aber nicht als Instrument der Regional- und Arbeitsmarktentwicklung, die ihrerseits Beiträge zu den Wachstumszielen leisten. Nach der Kommission sollen die Ziel-2-Mittel für Infrastrukturinvestitionen ergänzend zu den TEN-Projekten eingesetzt werden, um die Erreichbarkeit der Regionen zu erhöhen. Zwar kann der Anschluss an die TEN-Autobahn durchaus 
die Attraktivität einer Region verbessern, es ist aber dadurch ein Beitrag zur dauerhaften Beschäftigungssicherung nicht alleine gewährleistet. Hinzu kommt, dass die Mitgliedstaaten gerade im neuen Ziel 2 die Möglichkeit hätten, die Förderung auf ihre Wachstumszentren zu konzentrieren. Wenn im Sinne der Lissabon-Strategie als Ziele die Wachstumsförderung und die Steigerung der Wettbewerbsfähigkeit formuliert sind, dann mag dies eine effiziente Art des Mitteleinsatzes sein. Es mag auch Regionen geben, in denen die Wachstumszentren auf ihr Umland ausstrahlen. Fraglich ist jedoch, inwieweit man damit zu einer Abkoppelung der Entwicklungsgeschwindigkeiten europäischer Regionen beiträgt und langfristig negative Auswirkungen auf das gesamteuropäische Wachstum erzeugt. Unklar bleibt dabei auf jeden Fall, wie ein solcher Weg den Artikel I 58 erfüllen will.

Die Verordnungsvorschläge sehen insgesamt viel mehr politische Einflussmöglichkeiten für die Mitgliedstaaten vor, als es bisher der Fall war. Die bisherigen Einheitlichen Programmplanungsdokumente, die den Charakter eines Vertrags zwischen der Kommission und dem jeweiligen Mitgliedstaat hatten und die damit eine verbindliche Grundlage für die Durchführung der Förderung während einer siebenjährigen Förderperiode schufen, sollen durch die vom Ministerrat beschlossenen "Strategiepapiere« und die ergänzenden "Politikpapiere« jedes Mitgliedstaates ergänzt werden. Das wertet die politische Bedeutung des Rates auf, während die Kommission sich freiwillig eine untergeordnete Rolle zuschreibt. Es steht zu befürchten, dass die Strukturfonds zu einem Spielball des Rates werden. Da unklar ist, welche Rechtsqualität die "Strategiepapiere« haben sollen und ob sie für die gesamte Förderperiode gelten, könnten sie im Verlauf der Förderperiode durch den Rat verändert werden. Sie könnten damit im Rat zur "Verhandlungsmasse« werden.

Schließlich muss gefragt werden, welche Auswirkung diese geringe Verbindlichkeit der Planung auf das Partnerschaftsprinzip haben wird. Wenn die Mitgliedstaaten mehr politischen Spielraum für Durchführung und Planung erhalten, wird dies Auswirkungen auf die Mitwirkungsmöglichkeiten der Wirtschafts- und Sozialpartner haben. Im Extremfall würde ihre Beteiligung an Verbindlichkeit verlieren, wenn nicht sogar ganz eingestellt werden. Damit ginge ein innovatives Element der europäischen Förderung verloren: Der Dialog mit den Sozialpartnern in den Mitgliedstaaten und Region hat das erklärte Ziel, vor Ort die relevanten Akteure einzubeziehen, um mit ihnen auf die Region passende Gesamtwicklungskonzepte aufzustellen. Der europäischen Förderung ist es zu verdanken, dass sich in der deutschen Förderpraxis langsam eine Kultur von Partnerschaft herauszubilden beginnt. Diese beginnt auf der lokalen Ebene mit der Bildung von regionalen Bündnissen, die an konkreten Einzelprojekten arbeiten, und setzt sich in den Begleitstrukturen auf Landes- und Bundesebene fort, in denen die Wirtschafts- und Sozialpartner ihren Teil zur Abwicklung und Umsetzung der Förderung beitragen. Die Wirtschafts- und Sozialpartner nehmen diese Partnerschaft und diese Mitwirkungsmöglichkeiten sehr ernst, da sie ihnen die Möglichkeit geben, zu einer beschäftigungswirksamen Regionalentwicklung beizutragen. Die europäischen Institutionen haben den Nutzen des Partnerschaftsprinzips wiederholt anerkannt. Sie können sich auf Studien stützen, die die positiven Auswirkungen des Partnerschaftsprinzips durchaus belegen (vgl. Europäischer Wirtschafts- und Sozialausschuss 2003). 
Bisher galt der Grundsatz, dass der Mitteleinsatz aller Fonds insgesamt den Zielen der nachhaltigen Entwicklung im Sinne der Göteborg-Strategie, dem Gender Mainstreaming und der Unterstützung von KMU dienen sollte. Diese Querschnittsziele wurden weniger als quantitative Vorgaben, sondern vielmehr als qualitative Ausrichtungen verstanden. Die Förderung bekam eine Gesamtorientierung und die unterschiedlichen Fonds sollten nicht neben- oder gar gegeneinander eingesetzt werden. Bisher scheiterten die Querschnittsziele allerdings in der Förderpraxis an den wenig operationalen Anforderungen. Im Förderalltag der Verwaltungsbehörden kamen sie kaum vor. Mit dem neuerlichen Kommissionsvorschlag kommt die Kommission den nationalen Bürokratien entgegen, denen die Integration der Querschnittsziele in ihre Förderpraxis nicht gelungen ist. Insbesondere wird die nachhaltige Entwicklung von der Europäischen Kommission zwar erwähnt, seine Umsetzung aber nicht nachdrücklich gefordert. Das von der Europäischen Kommission vorgeschlagene "Monofonds«-Prinzip ist dann skeptisch zu beurteilen, wenn es der integrierten Förderung entgegensteht und zu einer »Versäulung« der unterschiedlichen Fördertatbestände führt.

\section{Ein kurzes Fazit}

Die EU ist am I. Mai 2004 größer geworden, die Nettozahler werden hartnäckiger - die Kommission stand vor der Quadratur des Kreises, mit weniger Geld mehr Strukturpolitik machen zu müssen. In Anbetracht dessen hat sie grundsätzlich das Prinzip der regionsbezogenen Strukturförderung und einer gebietsunabhängigen Beschäftigungsförderung beibehalten, gleichzeitig aber die nationalen Spielräume der Mitgliedstaaten erheblich ausgeweitet und die bisherige strukturpolitische Zielsetzung durch einen makroökonomischen Rahmen ergänzt. Damit muss die Strukturpolitik einen ganz neuen Spagat bewältigen, während sie gleichzeitig an Einheitlichkeit und Kontrollmöglichkeiten durch die Kommission verliert. Mit ihren Vorschlägen zur zukünftigen Strukturpolitik hat die Kommission eine neue Ära strukturpolitischer Maßnahmen in Europa eingeleitet, die aus beschäftigungspolitischer Sicht durchaus ambivalent zu beurteilen sind. Die Ausrichtung der Debatte am fiskalischen und nicht am inhaltlichen Aspekt, wie sie die Nettozahler betreiben, macht die Reformdebatte keineswegs einfacher. Es bleibt zu hoffen, dass die Wirkungsanalysen, die den Wachstumseffekt des bisherigen Systems klar belegen, in der weiteren Diskussion stärker beachtet werden.

\section{Literatur}

Axt, Heinz-Jürgen (2003): Was kostet die EU-Erweiterung?, in: WSI-Mitteilungen, Jg. 56, H. I, S. 3-9

Eltges, Markus (2003): Die Auswirkungen der EU-Osterweiterung auf die europäische Strukturpolitik, in: WSI-Mitteilungen, Jg. 56, H. I, S. IO-I5

Europäische Kommission (2004): Eine neue Partnerschaft für die Kohäsion. Konvergenz Wettbewerbsfähigkeit Kooperation. Dritter Bericht über den wirtschaftlichen und sozialen Zusammenhalt, Brüssel 
Europäische Wirtschafts- und Sozialausschuss (2003): Partnership for Implementing the Structural Funds, Brüssel

GEFRA, ESTI, IfS, MR, Ruhr-Universität, TraST (2004): Halbzeitbewertung des Gemeinschaftlichen Förderkonzeptes 2000-2006 (GFK) für den Einsatz der Strukturfonds in den neuen Bundesländern und im Ostteil Berlins, Münster et al.

Kommission der Europäischen Gemeinschaften (2004): Vorschlag für eine Verordnung des Rates mit allgemeinen Bestimmungen über den Europäischen Fonds für regionale Entwicklung, den Europäischen Sozialfonds und den Kohäsionsfonds, $\operatorname{KOM(2004)} 492$ endgültig, Brüssel

Petzold, Wolfgang (2004): Perspektiven der EU-Strukturpolitik, Vortrag gehalten am 2I.04.2004 in Berlin

Reissert, Bernd (2004): Der Einfluss von Europa, in: Gerlach, Frank/Ziegler, Astrid (Hg.), Neuere Herausforderungen der Strukturpolitik, Marburg, S. 257-282

Sapir André Group (2003): An Agenda for a Growing Europe - Making the EU Economic System Deliver, Brüssel

Ziegler, Astrid (2003a): Synopse wichtiger Positionen zur Reformdebatte der Europäischen Strukturpolitik nach 2006, WSI-Diskussionspapier, Nr. II4, Düsseldorf

Ziegler, Astrid (2003b): Die Europäische Strukturpolitik nach 2006. Anforderungen an ein neues Konzept der Europäischen Strukturfonds im Zeitraum von 2007 bis 2013, WSIDiskussionspapier, Nr. Iı6, Düsseldorf

\section{Kinderbetreuung: mehr als eine Investition in Erwerbspersonen und BeitragszahlerInnen der Zukunft Claudia Wiesner*}

\section{Die Kluft zwischen Reden und Handeln}

"In der Familienpolitik gibt es im Augenblick nichts Wichtigeres, als den Ausbau der Kinderbetreuung zu forcieren « - so Bundeskanzler Schröder in seiner Regierungserklärung 2002 (zitiert nach Belwe 2003). Die politischen Prioritäten in Deutschland, so sollte man meinen, wurden damit klar gesetzt. Die SPD reklamierte die "Lufthoheit über den Kinderbetten « für sich, die Unternehmerverbände entdeckten die Thematik, und nachdem sogar die als sehr konservativ geltende hessische CDU im Herbst 2004 ein Grundsatzprogramm verabschiedete, das explizit das Ideal der "Vereinbarkeit von Beruf und Familie« benennt, stoßen Forderungen nach Ganztagsschulen und besserer Kleinkindbetreuung inzwischen kaum

\footnotetext{
* Justus-Liebig-Universität Gießen.
} 